\title{
A Study of a Mobile Game on the Interrelationships of Technology Acceptance, Interpersonal Relation, Sense of Direction, and Information Literacy - A Case of Pokémon GO
}

\author{
Peng-Chun Lin, Hsin-Ke Lu, Yi-Hui Lin, and Wei-Huan Tsang
}

\begin{abstract}
The constant innovation of technology information has been prompting the development of mobile games. With the development, mobile games have an increasing influence on human beings. In June of 2016, Niantic Labs launched a mobile game called Pokémon GO, which led to a wave to play mobile games and stimulated the development of game industry. In addition, Pokémon GO was not only played for pleasure, but the police in Rotterdam, the Netherlands, also exploited the characteristic that Pokémon GO made players crowd in order to lower the crime rate. Additionally, Japan National Tourism Organization utilized Pokémon GO to increase the number of tourists by means of raising the probability of special pocket monsters showing up at specific spots. This study took a mobile game, Pokémon GO as a case and investigated the relationship among technology acceptance, interpersonal relation, sense of direction, and information literacy based on the model of technology acceptance (TAM).

The total number of the valid questionnaires which were collected in Taiwan was 306. The result showed that social influence and enjoyment had an impact on mobile game users' behavioral intention. The mobile game's ease of use perceived by the users had an effect on enjoyment of the mobile game. In addition, from users' self-evaluation, they thought this game improved their interpersonal relation, sense of direction, and information literacy. Future studies can center on users from different countries and explore design elements of mobile games, marketing strategies, and the impact on users' mental and physical state.
\end{abstract}

Index Terms-Mobile game, augmented reality, sense of direction, interpersonal relation, technology acceptance model (TAM), Pokémon GO.

\section{INTRODUCTION}

With a rapid advancement of network and mobile devices, the development of network service tends to diversify. One of the most important network services is mobile games. A survey conducted in Taiwan by Institute for Information Industry (2015) indicated that the number of users over 12 years old who use smart phones or tablet computers was over 16.04 million, accounting for 80 percent of the population over 12 years old in Taiwan [1]. Among the users of mobile devices in Taiwan, the number of game players was approximately 5.26 million, accounting for 65 percent of the

Manuscript received January 24, 2017; revised February 22, 2017

Peng-Chun Lin, Hsin-Ke Lu, and Yi-Hui Lin are with the Chinese Culture University, 231 Taipei, Taiwan (R.O.C.) (e-mail: pclin@sce.pccu.edu.tw, sklu@sce.pccu.edu.tw, yhlin@sce.pccu.edu.tw).

Wei-Huan Tsang is with the University of Sussex, BN1 9RH, United Kingdom (e-mail: wt83@sussex.uk). total game players [2]. According to Newzoo's (2005) statistics, Taiwan's overall revenue from game industry in 2015 was NT\$ 25.3 billion ranked 15th in the world [3]. Among the game industry in Taiwan, the output value of mobile games on Google Play and iOS systems was ranked 4th and 10th in the world respectively. On the whole, the aforementioned statistics revealed the high utilization rate of mobile games and potential of future development.

The advancement of technology is prompting the change of mobile game formats and creation of mobile game genres. In recent years, it has been a main stream of mobile games to adopt virtual reality (VR) and augmented reality (AR) technology. In 2012, the subsidiary company of Google, Niantic Lab, developed the first AR games, Ingress, which has been downloaded over 10 million times until 2016 in the world. Since the success of Ingress, game manufacturers have been developing a variety of AR games like Night Terrors, Father.io, Zombies Everywhere, etc. It has even been a new trend to develop AR games since Niantic Lab launched the AR game, Pokémon GO in 2016. In addition to being a popular AR game, the police at Vlaardingen, Rotterdam, the Netherlands, exploited the characteristic that Pokémon GO made players crowd by using the lure in the game to lower the crime rate [4]. Japan National Tourism Organization also utilized Pokémon GO to boost tourism by means of raising the probability of special pocket monsters showing up at specific spots [5]. Integrated with a variety of information systems, including networks, cameras, and Global Positioning System (GPS), Pokémon GO is an AR game for users to understand geographic environment and information technology. Therefore, the program of Business Information Technology at University of Salford has put Pokémon GO into its course design, and students in that program have to play the game to obtain the credit [6]. Many scholars of psychology also mentioned that Pokémon GO is able to help patients who suffer from anxiety and melancholia to a certain degree. What is more, Pokémon GO has the patients go outdoors, which is helpful for the patients to overcome crowd phobia and social communication disturbance [7].

This study explored users' technology acceptance of the mobile game, Pokémon GO, based on technology acceptance model, proposed by Davis (1989) [8]. It also integrated scales of interpersonal relation, sense of direction, and information literacy in order to explore whether users made progress in interpersonal relation, sense of direction, and information literacy. 


\section{LITERATURE REVIEW}

\section{A. Pokémon $G O$}

Pokémon GO was an AR game launched by Niantic Lab in June of 2016, and was available in Taiwan in August of 2016. The purpose of Pokémon GO was to encourage the public to walk outdoors for users to increase the amount of exercise and explore the outdoor attractions [9]. Therefore, Pokémon GO had been downloaded over 500 million times until October of 2016.

As for the application building techniques, Pokémon GO combined map simulation with GPS to ensure the position the users are at, and used techniques of AR to integrate the simulated game with physical environment. Therefore, on the screen of mobile devices, users can see the simulated Pokémons, i.e., the pocket monsters, in the real environment through the cameras. In Pokémon GO, the Pokémons will show up at any position on the map for a period of time. Before the period of time is due, all the players can capture the same Pokémon at the same spot. Therefore, there are many online communities exchanging information about Pokémons. This kind of non-competitive games is beneficial for users to share information. In fact, crowds could be seen at parks or scenic spots in each country, and these crowds communicated and interacted with each other face to face at the spot, which increases social interaction with the members of the crowds [9].

According to a survey conducted by Nielsen in Japan in September of 2016, Pokémon GO was a game suitable for all ages in that in each age group were a certain percentage of users of Pokémon GO. The total of the users over 40 years old exceeded 60 percent of all users. In addition, the age group with the most users was females whose age was between 18 and 29; the age groups with the second most users were males whose age was between 18 and 29 and males whose age was over 50 . As to the frequency of use, males over 50 years old and females between 40 and 49 years old turned on Pokémon GO more than twice a day and used it for more than 40 minutes, which indicated that users in middle age tended to be fully involved in Pokémon GO [10].

Lin (2016) mentioned that the reasons that made Pokémon GO such a popular game from a psychological perspective were as follows [11]:

1) Popular and proud: playing Pokémon GO is a conformity behavior. When playing Pokémon GO becomes a topic, individuals who play the game and are able to join the topic feel good and indirectly enhance self-esteem.

2) Occasional object: reinforcement is an important concept in the learning theory of operant conditioning. Reinforcement is a mechanism that can increase individuals' frequency of responses and reactions. In Pokémon Go, the random appearance of Pokémons and the random attainment of various items from Pokéstops, a stop that supplies users with props, serve a function as reinforcements.

3) King of Kingdom: users can move freely, fulfill their purposes, and attain the result by themselves in the virtual world.

4) Enchantment and Emotion excitation: in the process of playing Pokémon Go, positive emotion is activated by means of collecting Pokémons, and this positive emotion makes the action sustain.

5) Medal and Myth: the rewarding mechanics in Pokémon Go stir up users' interests. Furthermore, the characteristic of virtual games is that users can play a virtual role in the game, which makes the role to substitute the user to a stronger or better self in the game. Compared with users in real life, users in virtual world can become an ideal role in their mind easily.

6) Obsession and obliviousness: playing games involves classical conditioning. While everyone on the road is holding their smart phones and searching Pokémons, and the stimuli of Pokémon Go are surrounding, it is hard to resist turning on Pokémon Go and checking the surroundings through Pokémon Go, although you do not really want to play it.

7) Neighbor network: The purpose of Pokémon Go is to encourage the public to go outside, to observe the home environment that was neglected before, or to be willing to go to scenic spots that he or she has not been to. In fact, Pokémon Go has made many senior users attracted to gather at many scenic spots, like temples, parks, etc. The game involves real and visual situations, which are more likely to make individuals feel the link to the surrounding people, objects, and environment. Therefore, users may engage more in the game.

\section{B. Enjoyment}

Yeh (2010) stated that the reasons people like playing games are that games provide players with challenges and create a mental environment that players highly concentrate the attention on pursuing the goal of winning. In the process, players enter the flow state, achieving happiness and sense of fulfillment [12]. The research conducted by Rieber (1996) revealed that the nature of playing game is self-motivated and this activity makes players feel happy [13]. Therefore, the present study was based on technology acceptance model (TAM) and developed a proposed model. By using the proposed model, this study aimed to investigate whether the enjoyment of playing game had an effect on behavioral intention among players, and whether ease to use and social influence would affect enjoyment of players.

\section{Social Influence}

Oliver (1986) pointed out that the basic element to generate collective action is critical mass [14]. The unified theory of acceptance and use of technology (UTAUT) proposed by Venkatesh, Morris, Davis, and Davis (2003) also indicated that social influence would influence users' behavioral intention [15]. In addition, a study conducted by Chen (2013) indicated that social influence has an effect on behavioral intention of online game players [16]. Therefore, the current study explored whether social influence would have an impact on behavioral intention.

\section{Interpersonal Relation}

Interpersonal relation can be divided into two categories, interpersonal relation in real life and on the Internet. It has been found in many studies on online games that because users can create a virtual situation in the network environment and detach the virtual environment from the real 
one, introverted users or users who are not good at interpersonal communication are likely to seek emotional support in the network environment and are able to create an interpersonal relation different from that in the real environment. However, some relevant studies also proposed that if users have long been engaged in online games, it will lead to isolation and a barrier in the real-life interpersonal relation [17], [18].

The aim of Pokémon Go is not to establish a communication for online community, but to prompt users to go outside to exercise. Therefore, the current study centered on investigating the effect of Pokémon Go on users' interpersonal relation in real life.

\section{E. Sense of Direction}

Sense of direction refers to knowledge of the concept of orientation and direction, and spatial configuration [19]. Kozlowski and Bryant (1977) developed a scale on sense of direction, and in their research, they found that users who believed that they had good sense of direction tended to have a better performance on memorizing information of geography and direction [20]. In addition, the result in Cornell, Sorenson \& Mio's (2003) study also indicated that people with better sense of direction tended to make better use of environmental features, such as landmarks, to find their way and tended not to get lost easily [21]. Based on Kozlowski and Bryant (2007), Kato \& Takuechi (2003) further divided the concept of sense of direction into awareness of orientation and memory for usual spatial behavior [22].

The purpose of Pokémon Go is to encourage users to go outside; therefore, the gameplay is combined with GPS, which makes users be able to look for Pokémons based on the real map and orientation while playing the game. This study mainly discussed users' awareness of orientation, so only the questions of the dimension, i.e., awareness of orientation, were used to measure whether users had a significant improvement in sense of direction after using the game.

\section{F. Information Literacy}

Due to the rapid development of information technology, each country is putting emphasis on cultivating citizens' information literacy. Many scholars have pointed out that computer games can provide users with pleasant and exciting challenges, make mechanical exercises and drills become more lively and attractive, and reduce cognitive load. Chen (2002) indicated that it is an entry approach to use online games to cultivate the ability of application of computer hardware and software [19].

Computer Skills Foundation has developed an index of General Basic Information Technology Ability Test for Taiwanese citizens, which includes five fields: knowledge of information technology, use of information technology, data processing and analysis, understanding and application of Internet, and integration of information technology and humanistic quality. The design and mechanics of Pokémon Go integrated many important applications of technology through mobile devices. Therefore, this study investigated users' ability to use the important applications in Pokémon Go, such as augmented reality (AR), wireless network, GPS, and the concepts and application of mobile devices after users used Pokémon Go.

\section{RESEARCH METHOD}

This study used a quasi-experimental approach to investigate the learning outcomes of universities' students who were enrolled in a semester course that provided a basic competence to networks.

\section{A. The Proposed Technology Acceptance Model (TAM)}

The proposed model was based on technology acceptance model (TAM) proposed by Davis (1989) [8]. TAM was adopted to explore users' behavioral patterns of using a new information system. Moreover, the aim of TAM was to identify an effective model to describe the behavior of users when they use new information technology. In TAM, it was claimed that perceived usefulness and perceived ease of use would influence users' behavioral intention and further affect their actual system use. TAM was then extended and applied by many scholars to discuss online game acceptance among users [23], [24]. Hence, in the present study, TAM was taken as a fundamental model.

Based on the literature reviewed above, the TAM was modified to be suitable for the discussion of the TAM of mobile games. Perceived usefulness in the original model was replaced by enjoyment of gameplay and social influence was included to make a more extensive discussion. The model proposed in the present study is illustrated in the following figure (see Figure 1). The proposed model was adopted to investigate the relationships among social influence, enjoyment, ease of use, and behavioral intention.

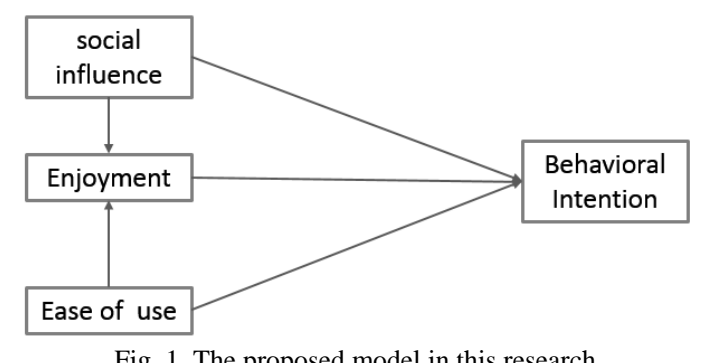

To analyze the data, the study adopted partial least square (PLS), which is an analytic technique to probe into or construct a predictive model. Especially for a causal model among latent variables, PLS is a better method than linear structural relations (LISREL). In addition, the advantage of PLS is that PLS is suitable for research with small sample size and is not restricted to the sampling distribution [25]. PLS also has good predictive and explanatory ability. Therefore, the current study adopted Smart PLS 3.0, software developed by Ringle, Wende, and Will (2005) to analyze the data. Bootstrap resampling method was used to repetitively draw 5000 samples to create an estimate parameter and make inference [26].

\section{B. Research Instrument}

This survey study recruited participants who had used Pokémon Go. The questionnaire was divided into three parts: demographic information, the proposed TAM, and 
self-assessment of interpersonal relation, sense of direction, and information literacy.

1) Demographic information: this part collected information about users' gender, age, and experience of playing Pokémon Go. Since the survey was conducted in October of 2016, and Pokémon Go had been just released only for three months, the answers for experience of playing Pokémon Go were divided into three categories, "less than a month", "one to two months", and "more than two months" in order to distinguish the users from new users, users with some experience, and experienced users.

2) The proposed TAM: this study modified the scale of TAM developed by Davis (1989) to collect data concerning "ease of use" and "behavioral intention". As for the other two dimensions, social influence and enjoyment, the questions were adapted from the scale of the unified theory of acceptance and use of technology (UTAUT), developed by Venkatesh et al. (2003), and were derived from the dimensions of social influence and performance expectancy in the original scale [15]. The questions were to explore players' feeling of enjoyment and social influence in gameplay [27]. The questionnaire adopted a five-point Likert scale, and the total number of the questions was 17.

3) Self-assessment of interpersonal relation, sense of direction, and information literacy: the questionnaire of interpersonal relation was adapted from that developed by Gong (2010) [28]. The total number of the questions was six. The scale of sense of direction was adapted from the dimension of awareness of orientation in the questionnaire developed by Kato and Takuechi (2003) [22]. The total number of the questions was seven. As for information literacy, the questions were based on the concept of information literacy proposed by Computer Skills Foundation. The total number of the questions was four. All the questions in this part used a five-point Likert scale.

\section{Procedures}

The study can be divided into two sessions, the pilot study and the main study. Before conducting the main study, a pilot study was carried out to test the validity and reliability of the questionnaires. In the pilot study, 30 participants were recruited, and based on the result of the statistical analysis, five question items were deleted. After the pilot study and deletion of the inappropriate questions, the questionnaires contained five questions for enjoyment, four for ease of use, two for behavioral intention, five for interpersonal relation, five for sense of direction, and four for information literacy. The total number of questions was 29. The values of Cronbach's $\alpha$ of each dimension were over .86, greater than .7, suggesting that the questions of this questionnaire had a good internal consistency. After the pilot study, the main study was conducted. Results and Discussion

The number of the questionnaires collected in the study was 309, among which 306 were valid. The total number of the male participants was $192(62.75 \%)$, and the total number of the female was $114(37.25 \%)$. The age of the participants ranged from 18 to 47 years old. The results of the examination of the proposed model are shown in the following figure (see Fig. 2).

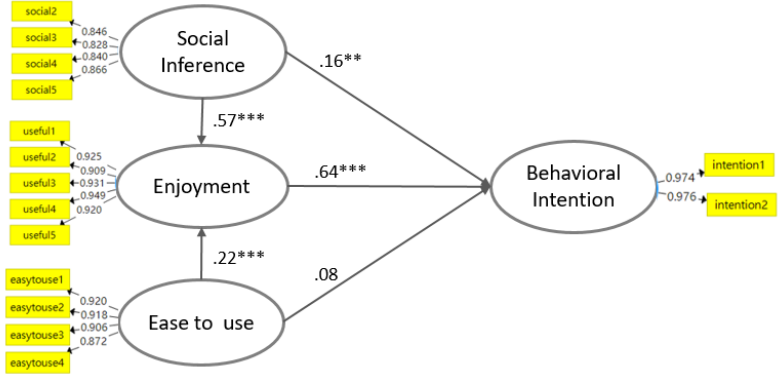

Fig. 2. The result of the path analysis.

The result shows that social influence had a significant relationship with enjoyment and behavioral intention. This result is consistent with other online game research, indicating that through the recommendation of users' friends, colleagues, or family, users tended to be more willing to use the games, their sense of identity and self-esteem may be promoted, and their feeling of enjoyment may be enhanced [14], [27]. Moreover, online games and mobile games are entertainment-oriented information system [23]. Therefore, if users have a better understanding of critical mass, they may be more willing to use online games.

Enjoyment was also related to users' behavioral intention, which is consistent with the research on online games [28]. As the feature of entertainment-oriented information systems was that users use the system voluntarily, so the more pleasant they can feel from the system, the more willing they are to use the system. In terms of ease of use, the results show that ease of use was significantly associated with enjoyment, which is consistent with the result of the original TAM that ease of use had a significant relationship with perceived usefulness [8]. When users use an information system, whether the information system is easy to use may affect users' opinions about usefulness. Because there are a variety of mobile games, if users cannot operate the game easily and smoothly, it will affect users' evaluation of the game and perceived enjoyment.

The only insignificant path was that ease of use did not significantly affect behavioral intention. A possibility may be that because Pokémon GO, a game suitable for all ages [10], is easy to operate and use, and there has been a wave to play the game, whether users feel the mobile game is easy to use would not affect users' behavioral intention.

In addition to the path analysis through partial least squares, users' experience of playing Pokémon GO was sent to two-step cluster analysis, which divided users into three groups, new users (124 users, 40.53\%), users with some experience (52 users, 26.14\%), and experienced users (102 users, $33.33 \%$ ). The data of self-assessments of interpersonal relation, sense of direction, and information literacy of the three groups were then sent to compare whether there was a significant difference among the three groups by ANOVA analysis. The results of ANOVA analysis revealed that there was a significant difference of the self-assessment of interpersonal relation, sense of direction, and information literacy among the three groups (see Table I).

Post Hoc analysis by Scheffe's method was performed. The results of post hoc analysis showed that the mean score of interpersonal relation for the experienced users $(\mathrm{M}=3.90)$ was significantly higher than that for the users with some 
experience $(M=3.64, p=.000<.001)$ and that for the new users $(M=3.28, p=.001<.01)$. Furthermore, as for interpersonal relation, the mean score for the users with some experience was significantly higher than that for the new users $(p=.025<.05)$. A possibility for this result could be that the experienced users spend more time on Pokémon GO, so they may have more opportunities to discuss or interact with the users around them, like family, friends, and those strangers using Pokémon GO at the scenic spots. Therefore, when the experienced users made the self-assessment, they might think their interpersonal relation was improved.

TABLE I: ANOVA ANALYSIS OF INTERPERSONAL RELATION, SENSE OF DIRECTION, AND INFORMATION LITERACY AMONG THE THREE GROUPS (NEW USERS, USERS WITH SOME EXPERIENCE, AND EXPERIENCED USERS)

\begin{tabular}{lcccc}
\hline \hline & df & Mean Square & F & Significance \\
\hline Interpersonal relation & 2 & 11.103 & 18.709 & $\mathbf{. 0 0 0}$ \\
Information Literacy & 2 & 6.137 & 7.094 & $\mathbf{. 0 0 1}$ \\
Sense of Direction & 2 & 7.954 & 10.008 & $\mathbf{. 0 0 0}$ \\
\hline \hline
\end{tabular}

In terms of information literacy, the mean score for the experienced users $(M=3.72)$ was significantly higher than that for the users with some experience $(M=3.39$, $p=.000<.001)$ and that for the new users (MD=3.26, $p=.019<.05)$. However, there was no significance between the users with some experience and the new users $(p=.31>.05)$. A possible reason may be that the experienced users have a stronger sense of identity toward Pokémon GO and spend more time studying how to use the devices to capture Pokémons precisely and easily. For example, the experienced users might try to understand what GPS is and how to adjust the function of WiFi to accurately position the location. Through the observation from the Internet community, the experienced users had discussed to a considerable extent the accuracy of positioning of mobile devices of each brand. Therefore, it can be speculated that the experienced users have a better understanding of the information technology applied to Pokémon GO, and feel that they have made a higher degree of improvement after play Pokémon GO than the other groups in the self-assessment.

With respect to sense of direction, the mean score for the experienced users $(M=3.58)$ was significantly higher than that for the users with some experience $(M=3.15$, $p=.000<.001)$ and that for the new users $(M=3.07$, $p=.001<.01)$. However, there was no significant difference between the users with some experience and the new users $(p=.51>.05)$. A possible reason is similar to the result of information literacy. Because the experienced users are more absorbed in the game, they tend to pay more attention to the position where Pokémons show up. For example, the experienced users used maps provided by a third party to discover and position where Pokémons were. This may also be the reason why the experienced users think that Pokémon $\mathrm{GO}$ is able to improve their sense of direction, but there was no significant difference between the rest of two groups.

\section{CONClusion AND Future StUdies}

This study on a mobile game, Pokémon GO, a global hit released in June of 2016, investigated the relationship among technology acceptance, interpersonal relation, sense of direction and information literacy. The result of the study showed that social influence and enjoyment had an effect on behavioral intention among mobile game users. This result of the study is consistent with the other studies. With regard to social influence, users' behavioral intention may be influenced by the surrounding people who recommended the game or by social trends. As for enjoyment, since mobile games are entertainment-oriented information systems, the enjoyment of the game is one of the most important factors to affect users' behavioral intention. In addition, other users' comments on the mobile game may also influence users' feelings toward it. For instance, In October of 2016, Niantic Lab released a Halloween activity on Pokémon GO, which led to an intense discussion and positive evaluation and brought in more users to use Pokémon GO.

This research also explored the difference of interpersonal relation, sense of direction, and information literacy among users with different experience of use. The result revealed that the experienced users topped the three groups of participants, i.e., the experienced users, the users with some experience, and the new users in the self-assessment of interpersonal relation, sense of direction, and information literacy. Statistical analysis showed that the self-assessments of interpersonal relation, sense of direction, and information literacy for the experienced users were significantly higher than the rest of the two groups. The possibility can be concluded that the experienced users are more dedicated to the game, so they spend more time investigating the information technology used in the game. Furthermore, with higher expectation to obtain the Pokémons or honors in the game, it takes the experienced users more time to investigate maps and orientation and where the scenic spots are.

As for interpersonal relation, although much research has indicated that those who get involved in online games tend to cause alienation and obstacles in the real interpersonal interaction and relation [17], [18]. However, because the aim of Pokémon GO is designed to prompt people to go outdoors and to scenic spots, Pokémon GO increases the opportunities for the users to interact with others in the public space instead. In addition, Pokémon GO also increases the opportunities to interact between friends, couples, and parents and children. The users' self-assessment in the present study also showed that the users of the three groups had positive feedback towards their interpersonal relation after using Pokémon GO.

The incessant advancement and innovation of Information technology prompts the development of mobile games, and the importance of mobile games has been being increased gradually. Moderate entertainment could relieve mental stress, promote interpersonal relation, and have users take more exercise. Nevertheless, indulging in games may have a negative impact on mental and physical state. Therefore, it is an important issue for users to have moderate body-mind balancing and good time management skills.

All the participants of the present study were Pokémon GO users in Taiwan. Future studies could further investigate the opinions of users from different countries and areas. Furthermore, it is another important issue to strengthen users' persistence and attraction to use mobile games. Future research can also explore marketing strategies of mobile 
games to promote and increase users' utilization rate and loyalty.

\section{REFERENCES}

[1] Institute for Information Industry. (2015). Foreseeing Innovative New Digiservice. [Online]. Available: http://www.find.org.tw/market_info.aspx?n_ID=8482

[2] Institute for Information Industry, 2016 Taiwan Games Market White Paper, 2016.

[3] Newzoo. (2015). The mobile games landscape in 2015 \& the power users who shaped it. [Online]. Available: https://newzoo.com/insights/articles/mobile-games-market-2015-freereport/

[4] Metro News. (2016). Politie Vlaardingen jaagt op criminelen met Pokémon. [Online]. Available: http://www.metronieuws.nl/nieuws/binnenland/2016/08/politie-vlaard ingen-jaagt-op-criminelen-met-pokemon

[5] Japhub. (2016). Japan tourism board and Niantic lab strategic alliance to revitalize the Japanese tourism industry. [Online]. Available: http://japhub.com/?c=4013

[6] D. Mail. (2016). Now university offers a degree in Pokemon: Lecturer says the game makes the business information technology course more 'accessible'. [Online]. Available: http://www.dailymail.co.uk/sciencetech/article-3817771/Now-univers ity-offers-degree-Pokemon-Lecturer-says-game-makes-business-infor mation-technology-course-accessible.html

[7] Seehwa. (2016). Psychologist: Pokémon Go can help patients with mental disorders. [Online] Available: http://news.seehua.com/?p=192669

[8] F. D. Davis, "Perceived usefulness, perceived ease of use and end-user acceptance of information technology," MIS Quarterly, vol. 13, pp. 318-339, 1989.

[9] A. Griffin, Pokémon Go: Trainer who became first in UK to catch 'em all lost two stone while doing so, 2016.

[10] Nielsen. (2016). Nielson Mobile NetView. [Online]. Available: https://www.webprofessional.jp/looked-about-the-game-application-2 01609/

[11] C. C. Lin, An Analysis of the Pokémon Go from Psychology, 2016.

[12] S. Y. Yeh, The Introduction of Digital Game Design, Taipei: Gotop, 2010.

[13] L. P. Rieber, "Seriously considering play: Designing interactive learning environments based on the blending of microworlds, simulations, and games," Educational Technology Research and Development, vol. 44, no. 2, pp. 43-58, 1996.

[14] M. Oliver, Understanding Disability: From Theory to Practice, London, Macmillan, 1986.

[15] V. Venkatesh, M. G. Morris, G. B. Davis, and F. D. Davis, "User acceptance of information technology: Toward a unified view," MIS Quarterly, vol. 27, pp. 425-478, 2003.

[16] L. W. Chen, A Study of Social Networking Site Affect to Participate in An Online Game, Taichung: ITU, 2013.

[17] J. Morahan-Martin and P. Schumacher, "Incidence and correlates of pathological Internet use among college students," Computers in Human Behavior, vol. 16, pp.13-29, 2000.

[18] S. F. Chen and M. N. Yu, "A survey study on the six graders' computer games addiction and the interpersonal relationship taking Kaohsiung city, Kaohsiung county and Pingtung county as examples," Journal of Educational Research and Development, vol. 5, no. 4, pp. 219-244, 2009.

[19] W. C. Chen, "My view on the adolescents' culture of internet café," Counseling \& Guidance, vol. 198, pp. 53-55, 2002.

[20] L. T. Kozlowski and K. J. Bryant, "Sense of direction, spatial orietation, and cognitive maps," Journal of Experimental Psychology, vol. 3, pp. 590-598, 1977.
[21] E. H. Cornell, A. Sorenson, and T. Mio, "Human sense of direction an wayfinding," Annals of the Association of American Geographers, vol. 93, no. 2, pp. 399-425, 2003.

[22] Y. Kato and Y. Takeuchi, "Individual differences in wayfinding strategies," Journal of Environment Psychology, vol. 23, pp. 171-188, 2003.

[23] C. L. Hsu and H. P. Lu, "Why do people play on-line games? An extended TAM with social influences and flow experience," Information \& Management, vol. 41, no. 7, pp. 853-868, 2004.

[24] A. Yusoff, R. Crowder, and L. Gilbert, "Validation of serious games attributes using the technology acceptance model," in Proc. 2010 Second International Conference on Games and Virtual Worlds for Serious Applications(VS-GAMES),, pp. 45-51, 2010.

[25] D. M. Pirouz, An Overview of Partial Least Squares, 2006.

[26] C. M. Ringle, S. Wende, and A. Will, SmartPLS-Version 2.0. Germany: University at Hamburg, 2005.

[27] H. K. Lu, P. C. Lin, and Y. C. Lin, "A study of the factors affecting the purchase intention on mobile game apps," Journal of Advances in Information Technology, vol. 7, no. 4, pp. 239-244, 2016.

[28] H. L. Gong, A Study toward the Internet Usage Behaivor, Internet Addiction, and Real Relationship and Network Relationship for College Students, Taiwan: National Dong Hwa University, 2010.

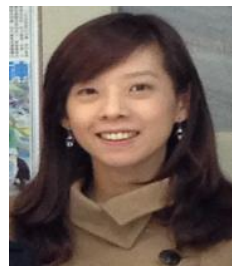

Peng-Chun Lin is an assistant professor of the Department of Information Management in Chinese Culture University and the Ph.D. candidate of Information Education Research Institute of National Taiwan Normal University. She is also the chief officer of the International Information Certification Cooperation Center at Chinese Culture University in Taiwan. Her academic interests focus on e-learning, network community development, application of simulation learning and recently on optimal network management and corporate structure.

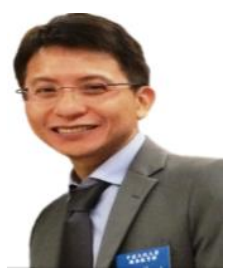

Hsin-Ke Lu is the director of information managemen (master programs for working learners) and the CEO of School of Continuing Education at Chinese Culture University in Taiwan. $\mathrm{He}$ is the chairman of the Association of Continuing Education of Colleges and Universities in Taiwan and the Chairman of Cisco Networking Academy. He is also a holder of Open Group Certification for Enterprise Architecture and Certification in the Governance of Enterprise IT. His academic interests focus on e-learning, corporate structure, lifelong learning information system planning (enterprise architecture).

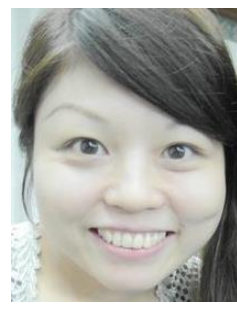

Yi-Hui Lin is the postdoctoral fellow in School of Continuing Education, Chinese Culture University, Taipei, Taiwan. She received her Ph.D. degree from the Department of Human Development and Family Studies at National Taiwan Normal University in August 2013. She also holds an Ed.M in learning and instruction from State University of New York at Buffalo. Her research interests include game-based learning in early childhood education, early science education, early conceptions of learning, learning and instruction in early childhood education.

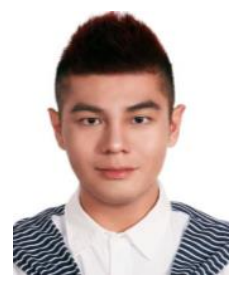

Wei-Huan Tsang graduated from the Department of Geography, National Taiwan University. $\mathrm{He}$ is a master student in University of Sussex now and his research interests include e-learning, social networking and the research of sexual dissidence. 